\title{
Supersymmetry Breaking in Warped Geometry
}

\author{
Kiwoon Choi \\ Department of Physics, Korea Advanced Institute of Science and Technology, \\ Daejeon 305-701, Korea \\ E-mail: «choi@hep.kaist.ac.kr \\ Do Young Kim \\ Department of Physics, Korea Advanced Institute of Science and Technology, \\ Daejeon 305-701, Korea \\ E-mail: kimdoyoung@hep.kaist.ac.kr \\ lan-Woo Kim \\ Department of Physics, Korea Advanced Institute of Science and Technology, \\ Daejeon 305-701, Korea \\ E-mail: iwkim@hep.kaist.ac.kr

\section{Tatsuo Kobayashi} \\ Department of Physics, Kyoto University, Kyoto 606-8502, Japan \\ E-mail: kobayash@gauge.scphys.kyoto-u.ac.jp
}

\begin{abstract}
We examine the soft supersymmetry breaking parameters in supersymmetric theories on a slice of $\mathrm{AdS}_{5}$ which generate the hierarchical Yukawa couplings by dynamically localizing the bulk matter fields in extra dimension. Such models can be regarded as the AdS dual of the recently studied 4-dimensional models which contain a supersymmetric CFT to generate the hierarchical Yukawa couplings. In such models, if supersymmetry breaking is mediated by the bulk radion superfield and/or some brane chiral superfields, potentially dangerous flavor-violating soft parameters can be naturally suppressed, thereby avoiding the SUSY flavor problem. We present some models of radion-dominated supersymmetry breaking which yield a highly predictive form of soft parameters in this framework.
\end{abstract}

Keywords: Supersymmetry Breaking, Field Theories in Higher Dimension, Supersymmetric Effective Theories. 


\section{Contents}

1. Introduction 1

2. Yukawa couplings and Soft parameters in Supersymmetric AdS $_{5}$ model 2

3. Radion-dominated Scenario 9

4. Conclusion 13

\section{Introduction}

Warped extra dimension can provide a rationale for various hierarchical structures in particle physics. For instance, in 5-dimensional (5D) theory on a slice of $\mathrm{AdS}_{5}$ with $\mathrm{AdS}$ curvature $k$ and orbifold radius $R$, the small warp factor $e^{-\pi k R}$ can generate the huge hierarchy between the $4 \mathrm{D}$ Planck scale $\left(M_{P l} \sim 10^{18} \mathrm{GeV}\right)$ and the weak scale $\left(M_{W} \sim 10^{2}\right.$ $\mathrm{GeV}$ ) [1], and/or the hierarchical quark and lepton masses [2, 3], and/or small neutrino masses $₫$. The underlying dynamics for small $M_{W} / M_{P l}$ and small fermion masses was the localization of gravity and fermion zero modes in extra dimension.

In this paper, we wish to examine soft supersymmetry (SUSY) breaking parameters in models with warped extra dimension in which the hierarchical Yukawa couplings are generated by localizing the bulk matter fields in extra dimension [5]. To this end, we consider supersymmetric 5D theory on a slice of $\mathrm{AdS}_{5}$ in which all quark and lepton fields arise from 5D bulk hypermultiplets [2]. The graviphoton of the model can be gauged appropriately to have nonzero bulk cosmological constant and hypermultiplet masses which would make the gravity zero mode localized at one boundary $(y=0)$ and the matter zero modes localized at any of the two boundaries $(y=0$ or $\pi)$. In this type of models, the small warp factor and the small Yukawa couplings have a common origin, i.e. the dynamical localization of zero modes associated with the gauging of graviphoton. As a result, the small Yukawa couplings are typically given by $y \sim e^{-c \pi k R}$ where $c$ is a constant of order unity, which would require that the radion is stabilized at a value giving the warp factor: $e^{-\pi k R} \approx 10^{-2}-10^{-5}$ and the corresponding Kaluza-Klein (KK) scale: $M_{K K} \approx k e^{-\pi k R} \sim 10^{16}-10^{13} \mathrm{GeV}$. This type of models can be regarded as the AdS dual of the recently studied 4D models [6] which contain a supersymmetric CFT sector generating the Yukawa hierarchy through renormalization group evolution.

It has been noted that supersymmetric CFT dynamics can provide also a mechanism to suppress dangerous flavor-violating soft parameters [6]. This suggests that a similar suppression of flavor-violation can take place in $\mathrm{AdS}_{5}$ models also. As we will see, flavorviolating soft parameters in $\mathrm{AdS}_{5}$ models are indeed suppressed under a reasonable assumption on SUSY breaking. Furthermore, if SUSY is broken by the $F$-term of the radion 
superfield or equivalently by the Scherk-Schwarz mechanism [7, 8], the model leads to a concrete prediction on soft parameters which can be tested by future experiments. In this paper, we first discuss general forms of soft parameters in supersymmetric $\mathrm{AdS}_{5}$ model, and later present some models of radion-dominated SUSY breaking in $\mathrm{AdS}_{5}$ which can pass all constraints on flavor-violation without severe fine tuning of parameters. Most of our results on the Yukawa couplings and soft parameters can be applied also to the models with dynamically localized bulk matter fields in flat extra dimension. For this, one can simply take a limit that the AdS curvature becomes zero, while the hypermultiplet masses remain to be nonzero.

\section{Yukawa couplings and Soft parameters in Supersymmetric $\mathbf{A d S}_{5}$ model}

To proceed, let us consider a generic 5D gauge theory coupled to the minimal 5D supergravity (SUGRA) on $S^{1} / Z_{2}$. The action of the model is given by [2, 9, 10]

$$
\begin{aligned}
S=\int d^{5} x \quad & \sqrt{-G}\left[\frac{1}{2}\left(\mathcal{R}+\bar{\Psi}_{M}^{i} \gamma^{M N P} D_{N} \Psi_{i P}-\frac{3}{2} C_{M N} C^{M N}+12 k^{2}\right)\right. \\
& +\frac{1}{g_{5 a}^{2}}\left(-\frac{1}{4} F^{a M N} F_{M N}^{a}+\frac{1}{2} D_{M} \phi^{a} D^{M} \phi^{a}+\frac{i}{2} \bar{\lambda}^{a i} \gamma^{M} D_{M} \lambda_{i}^{a}\right) \\
& \left.+\left|D_{M} h_{I}^{i}\right|^{2}+i \bar{\Psi}_{I} \gamma^{M} D_{M} \Psi_{I}+i c_{I} k \epsilon(y) \bar{\Psi}_{I} \Psi_{I}+\ldots\right]
\end{aligned}
$$

where $\mathcal{R}$ is the $5 \mathrm{D}$ Ricci scalar, $\Psi_{M}^{i}(i=1,2)$ are the symplectic Majorana gravitinos, $C_{M N}=\partial_{M} B_{N}-\partial_{N} B_{M}$ is the graviphoton field strength, and $y$ is the 5 th coordinate with a fundamental range $0 \leq y \leq \pi$. Here $\phi^{a}, A_{M}^{a}$ and $\lambda^{i a}$ are $5 \mathrm{D}$ scalar, vector and symplectic Majorana spinors constituting a $5 \mathrm{D}$ vector multiplet, $h_{I}^{i}$ and $\Psi_{I}$ are $5 \mathrm{D}$ scalar and Dirac spinor constituting the $I$-th hypermultiplet with kink mass $c_{I} k \epsilon(y)$. The AdS curvature $k$ and the kink mass $c_{I} k$ are related with the gauging of graviphoton as indicated by the following covariant derivatives [9, 11]:

$$
\begin{aligned}
D_{M} h_{I}^{i} & =\partial_{M} h_{I}^{i}-i\left(\frac{3}{2}\left(\sigma_{3}\right)_{j}^{i}-c_{I} \delta_{j}^{i}\right) k \epsilon(y) B_{M} h_{I}^{j}+\ldots, \\
D_{M} \Psi_{I} & =\partial_{M} \Psi_{I}+i c_{I} k \epsilon(y) B_{M} \Psi_{I}+\ldots, \\
D_{M} \lambda^{a i} & =\partial_{M} \lambda^{a i}-i \frac{3}{2}\left(\sigma_{3}\right)_{j}^{i} k \epsilon(y) B_{M} \lambda^{a j}+\ldots,
\end{aligned}
$$

where the ellipses stand for other gauge couplings. Note that we set the 5D Planck mass $M_{5}=1$ and all dimensionful parameters, e.g. the 5D gauge coupling $g_{5 a}$ and the AdS curvature $k$, are defined in this unit. Although not required within 5D SUGRA, it is not unreasonable to assume that the graviphoton charges $\left(c_{I}\right)$ are quantized in an appropriate unit, i.e. $c_{I} / c_{J}$ are rational numbers, which we will adopt throughout this paper. We note that in CFT view, $c_{I}$ are related to the anomalous dimensions $\gamma_{I}$ of chiral operators built from CFT-charged superfields [6]. At a superconformal fixed point, $\gamma_{I}$ are determined simply by group theory factors, so are quantized.

With appropriate values of the brane cosmological constants at the orbifold fixed points $(y=0, \pi)$, the ground state geometry of the action (2.1) is given by a slice of $\mathrm{AdS}_{5}$ having 
the following form of $5 \mathrm{D}$ metric:

$$
G_{M N} d x^{M} d x^{N}=e^{-2 k R y} \eta_{\mu \nu} d x^{\mu} d x^{\nu}+R^{2} d y^{2}
$$

It is convenient to write the $5 \mathrm{D}$ action (2.1) in $N=1$ superspace [12, 0, 13]. For the 5D SUGRA multiplet, we keep only the radion superfield

$$
T=\left(R+i B_{5}, \frac{1}{2}\left(1+\gamma_{5}\right) \Psi_{5}^{i=2}\right)
$$

and replace other fields by their vacuum expectation values. For the $5 \mathrm{D}$ vector multiplets and hypermultiplets, one needs appropriate $R$ and $B_{5}$-dependent field redefinitions to construct the corresponding $N=1$ superfields [7, 11]. After such field redefinition, the relevant piece of the action is given $b^{1}$ [12, []

$$
\begin{aligned}
& \int d^{5} x {\left[\int d^{4} \theta \frac{T+T^{*}}{2}\left(\hat{H}_{I}^{*} \hat{H}_{I}+\hat{H}_{I}^{c *} \hat{H}_{I}^{c}\right)+\left\{\int d^{2} \theta \frac{1}{4 g_{5 a}^{2}} T W^{a \alpha} W_{\alpha}^{a}+h . c .\right\}\right] } \\
&=\int d^{5} x {\left[\int d^{4} \theta \frac{T+T^{*}}{2}\left(e^{\left(\frac{1}{2}-c_{I}\right)\left(T+T^{*}\right) k|y|} H_{I}^{*} H_{I}+e^{\left(\frac{1}{2}+c_{I}\right)\left(T+T^{*}\right) k|y|} H_{I}^{c *} H_{I}^{c}\right)\right.} \\
&\left.+\left\{\int d^{2} \theta \frac{1}{4 g_{5 a}^{2}} T W^{a \alpha} W_{\alpha}^{a}+\text { h.c. }\right\}\right],
\end{aligned}
$$

where $W_{\alpha}^{a}$ is the chiral spinor superfield for the vector superfield $\mathcal{V}^{a}$ containing $\left(A_{\mu}^{a}, \lambda^{a}\right)$ with $\lambda^{a}=\frac{1}{2}\left(1-\gamma_{5}\right) \lambda^{a 1}, H_{I}$ and $H_{I}^{c}$ are chiral superfields containing $\left(h_{I}^{1}, \psi_{I}\right)$ and $\left(h_{I}^{2 *}, \psi_{I}^{c}\right)$, respectively, with $\psi_{I}=\frac{1}{2}\left(1-\gamma_{5}\right) \Psi_{I}, \bar{\psi}^{c}{ }_{I}=\frac{1}{2}\left(1+\gamma_{5}\right) \Psi_{I}$. Here we consider two superfields bases for hypermultiplets, $\left(\hat{H}_{I}, \hat{H}_{I}^{c}\right)$ and $\left(H_{I}, H_{I}^{c}\right)$, which are related to each other by

$$
\begin{aligned}
\hat{H}_{I} & =e^{\left(\frac{1}{2}-c_{I}\right) T k|y|} H_{I}, \\
\hat{H}_{I}^{c} & =e^{\left(\frac{1}{2}+c_{I}\right) T k|y|} H_{I}^{c} .
\end{aligned}
$$

As the theory is orbifolded by $Z_{2}: y \rightarrow-y$, all $5 \mathrm{D}$ fields should have a definite boundary condition under $Z_{2}$. The 5D SUGRA multiplet is assumed to have the standard boundary condition leaving the $4 \mathrm{D} N=1 \mathrm{SUSY}$ unbroken. To give a massless $4 \mathrm{D}$ gauge multiplet, the vector superfield $\mathcal{V}^{a}$ is required to be $Z_{2}$-even:

$$
\mathcal{V}^{a}(-y)=\mathcal{V}^{a}(y)
$$

On the other hand, the hypermultiplet can have any $Z_{2}$-boundary condition:

$$
\begin{aligned}
& H_{I}(-y)=z_{I} H_{I}(y), \\
& H_{I}^{c}(-y)=-z_{I} H_{I}(y),
\end{aligned}
$$

where $z_{I}= \pm 1$, and then a massless $4 \mathrm{D}$ chiral superfield $Q_{I}$ originates from either $H_{I}$ $\left(z_{I}=1\right)$ or $H_{I}^{c}\left(z_{I}=-1\right)$.

\footnotetext{
${ }^{1}$ Note that our $H_{I}$ and $H_{I}^{c}$ differ from $\left[7\right.$ which is related to ours by $H_{I} \rightarrow e^{-\left(\frac{3}{2}-c_{I}\right) T k|y|} H_{I}$ and $H_{I}^{c} \rightarrow e^{-\left(\frac{3}{2}+c_{I}\right) T k|y|} H_{I}^{c}$.
} 
In the superfield basis of $\left(H_{I}, H_{I}^{c}\right)$, the $I$-th $4 \mathrm{D}$ superfield $Q_{I}$ appears as the $y$ independent mode of $\left(H_{I}, H_{I}^{c}\right)$. However in another superfield basis of $\left(\hat{H}_{I}, \hat{H}_{I}^{c}\right)$ for which the $5 \mathrm{D}$ action (2.4) has a standard form of Kähler metric, the wavefunction of $Q_{I}$ appears to be localized near at one of the orbifold fixed points:

$$
\hat{H}_{I} \sim e^{-q_{I} T k|y|} \quad\left(z_{I}=1\right) \quad \text { or } \quad \hat{H}_{I}^{c} \sim e^{-q_{I} T k|y|} \quad\left(z_{I}=-1\right)
$$

where

$$
q_{I}=z_{I} c_{I}-\frac{1}{2}
$$

So, $Q_{I}$ is localized near at $y=0$ if $q_{I}>0$, while localized at $y=\pi$ if $q_{I}<0$. As is well known, such localization of matter zero modes can generate naturally the hierarchical Yukawa couplings. It is in fact possible to achieve the dynamical localization of matter zero modes in flat extra dimension [14, 12]. However in 5D SUGRA context, the localization of matter zero modes has the same origin as the localization of gravity zero mode, i.e. the gauging of graviphoton as (2.2). In this sense, warped extra dimension with localized $4 \mathrm{D}$ gravity can be considered as a more natural ground than flat extra dimension for the dynamical localization of matter zero modes. Furthermore, the localization of matter zero modes in $\mathrm{AdS}_{5}$ has an intrepretation in terms of supersymmetric $4 \mathrm{D} \mathrm{CFT}$ as the renormalization group evolution at superconformal fixed point [6].

In addition to the bulk action (2.4), there can be brane actions at the fixed points $y=0, \pi$. The general covariance requires that the $4 \mathrm{D}$ metric in brane action should be the $4 \mathrm{D}$ component of the $5 \mathrm{D}$ metric at fixed point. Using the general covariance and also the $R$ and $B_{5}$-dependent field redefinitions which have been made to construct $N=1$ superfields, one can easily find the $T$-dependence of brane actions [7, 15, 16]. For instance, the brane actions which would be relevant for Yukawa couplings and soft parameters are given by ${ }^{2}$

$$
\begin{aligned}
S_{\text {brane }}= & \int d^{5} x\left[\delta ( y ) \left\{\int d^{4} \theta L_{I \bar{J}}\left(Z, Z^{*}\right) \Phi_{I} \Phi_{J}^{*}\right.\right. \\
& \left.+\left(\int d^{2} \theta \frac{1}{4} \omega_{a}(Z) W^{a \alpha} W_{\alpha}^{a}+\lambda_{I J K}(Z) \Phi_{I} \Phi_{J} \Phi_{K}+\text { h.c }\right)\right\} \\
& +\delta(y-\pi)\left\{\int d^{4} \theta e^{-\left(q_{I} \pi k T+q_{J} \pi k T^{*}\right)} L_{I \bar{J}}^{\prime}\left(Z^{\prime}, Z^{\prime *}\right) \Phi_{I} \Phi_{J}^{*}\right. \\
& \left.\left.+\left(\int d^{2} \theta \frac{1}{4} \omega_{a}^{\prime}\left(Z^{\prime}\right) W^{a \alpha} W_{\alpha}^{a}+e^{-\left(q_{I}+q_{J}+q_{K}\right) \pi k T} \lambda_{I J K}^{\prime}\left(Z^{\prime}\right) \Phi_{I} \Phi_{J} \Phi_{K}+\text { h.c }\right)\right\}\right]
\end{aligned}
$$

where $Z$ and $Z^{\prime}$ denote generic $4 \mathrm{D}$ chiral superfields living only on the brane at $y=0$ and $y=\pi$, respectively, and

$$
\Phi_{I}=H_{I} \quad\left(z_{I}=1\right) \quad \text { or } \quad \Phi_{I}=H_{I}^{c} \quad\left(z_{I}=-1\right)
$$

Here $L_{I \bar{J}}\left(L_{I \bar{J}}^{\prime}\right)$ are generic hermitian functions of $Z$ and $Z^{*}\left(Z^{\prime}\right.$ and $\left.Z^{\prime *}\right)$, and $\omega_{a}$ and $\lambda_{I J K}$ $\left(\omega^{\prime}\right.$ and $\left.\lambda_{I J K}^{\prime}\right)$ are generic holomorphic functions of $Z\left(Z^{\prime}\right)$.

\footnotetext{
${ }^{2}$ The chiral anomaly of the $R$ and $B_{5}$-dependent field redefinition induces $T$-dependent pieces in $\omega_{a}$ and $\omega_{a}^{\prime}$ [1]. But they are loop-suppressed and not very relevant for the discussion in this paper.
} 
If there is no light gauge-singlet 5D field other than the minimal 5D SUGRA multiplet, SUSY breaking would be mediated by the radion superfield $T$, and/or the brane superfields $Z, Z^{\prime}$, and/or the 4D SUGRA multiplet which always participate in the mediation of SUSY breaking through the conformal anomaly [17]. The relative importance of anomalymediation (compared to radion-mediation) depends on the details of radion stabilization. Although anomaly-mediation can give an important (or even dominant) contribution to soft parameters in some special case [18, in the class of models under consideration, the contributions from anomaly-mediation are generically negligible as they are further suppressed by warp factor as well as by the loop factor [19]. So in this paper, we will focus on the soft SUSY breaking parameters induced by the $F$-terms of $T$ and $Z, Z^{\prime}$.

The 4D Yukawa couplings and soft parameters can be most easily studied by constructing the effective action of massless $4 \mathrm{D}$ superfields. As we have noted, in the superfield basis of $\left(\mathcal{V}^{a}, H_{I}, H_{I}^{c}\right)$, the $y$-independent constant modes correspond to the massless $4 \mathrm{D}$ superfields of the model. Let $V^{a}$ denote the constant mode of $\mathcal{V}^{a}$, and $Q_{I}$ to be the constant mode of $H_{I}\left(z_{I}=1\right)$ or of $H_{I}^{c}\left(z_{I}=-1\right)$. Here we will assume that all visible 4D gauge and matter fields are in $\left\{V^{a}, Q_{I}\right\}$, and examine their Yukawa couplings and soft SUSY breaking parameters when the SUSY breaking is mediated by $T$ and/or $Z, Z^{\prime}$. Those Yukawa couplings and soft parameters at the Kaluza-Klein scale $M_{K K} \approx k e^{-\pi k R}$ can be evaluated from the $4 \mathrm{D}$ effective action which can be written as

$$
\left[\int d^{4} \theta Y_{I \bar{J}} Q_{I} Q_{J}^{*}\right]+\left[\int d^{2} \theta\left(\frac{1}{4} f_{a} W^{a \alpha} W_{\alpha}^{a}+\tilde{y}_{I J K} Q_{I} Q_{J} Q_{K}\right)+\text { h.c. }\right],
$$

where $Y_{I \bar{J}}$ are hermitian wave function coefficients, $f_{a}$ are holomorphic gauge kinetic functions, and $\tilde{y}_{I J K}$ are holomorphic Yukawa couplings. Using (2.4) and (2.8), we find

$$
\begin{aligned}
Y_{I \bar{J}} & =\frac{1}{q_{I} k}\left(1-e^{-q_{I} \pi k\left(T+T^{*}\right)}\right) \delta_{I J}+L_{I \bar{J}}\left(Z, Z^{*}\right)+\frac{L_{I \bar{J}}^{\prime}\left(Z^{\prime}, Z^{\prime *}\right)}{e^{\left(q_{I} \pi k T+q_{J} \pi k T^{*}\right)}}, \\
f_{a} & =\frac{2 \pi}{g_{5 a}^{2}} T+\omega_{a}(Z)+\omega_{a}^{\prime}\left(Z^{\prime}\right), \\
\tilde{y}_{I J K} & =\lambda_{I J K}(Z)+\frac{\lambda_{I J K}^{\prime}\left(Z^{\prime}\right)}{e^{\left(q_{I}+q_{J}+q_{K}\right) \pi k T}} .
\end{aligned}
$$

Note that 5D SUSY in bulk enforces that the Yukawa couplings of $Q_{I}$ originate entirely from the brane action (2.8).

It is straightforward to compute soft parameters for generic forms of $L_{I \bar{J}}, L_{I \bar{J}}^{\prime}[19$. Here we will consider the case with

$$
\begin{aligned}
L_{I \bar{J}}\left(Z, Z^{*}\right) & =-\kappa_{I \bar{J}} Z Z^{*}, \\
L_{I \bar{J}}^{\prime}\left(Z^{\prime}, Z^{\prime *}\right) & =-\kappa_{I \bar{J}}^{\prime} Z^{\prime} Z^{\prime *},
\end{aligned}
$$

where $\kappa_{I \bar{J}}$ and $\kappa_{I \bar{J}}^{\prime}$ are generic constants of order one. However, we will keep $\omega_{a}$ and $\lambda_{I J K}$ to be generic holomorphic functions of $Z$, and $\omega_{a}^{\prime}$ and $\lambda_{I J K}^{\prime}$ to be generic holomorphic functions of $Z^{\prime}$. The results for more general forms of $L_{I \bar{J}}$ and $L_{I \bar{J}}^{\prime}$ will be presented elsewhere [19]. In regard to the suppression of flavor-violating soft parameters, those general results show a similar behavior as our case. For simplicity, we further assume that

$$
\langle Z\rangle \ll 1, \quad\left\langle Z^{\prime}\right\rangle \ll 1,
$$


in the unit with $M_{5}=1$, so

$$
\left\langle L_{I \bar{J}}\right\rangle \ll 1, \quad\left\langle L_{I \bar{J}}^{\prime}\right\rangle \ll 1 .
$$

In regard to SUSY breaking, we consider the general case in which any of the $F$-components of $T, Z$ and $Z^{\prime}$ can be the major source of SUSY breaking. Note that here we are not concerned with the dynamial origin of those $F$-components, but with the resulting soft parameters of visible fields for generic values of the $F$-components.

Let $y_{I J K}, M_{a}, m_{I \bar{J}}^{2}$, and $A_{I J K}$ denote the Yukawa couplings, gaugino masses, soft scalar masses, trilinear scalar couplings, respectively, for the canonically normalized matter superfields $Q_{I}=\phi^{I}+\theta \psi^{I}+\theta^{2} F^{I}$ and gauginos $\lambda^{a}$ which are renormalized at $M_{K K}$ :

$$
\frac{1}{2} y_{I J K} \phi_{I} \psi_{J} \psi_{K}-\frac{1}{2} M_{a} \lambda^{a} \lambda^{a}-\frac{1}{2} m_{I \bar{J}}^{2} \phi^{I} \phi^{J *}-\frac{1}{6} A_{I J K} \phi^{I} \phi^{J} \phi^{K}+\text { h.c. }
$$

One then finds from (2.10):

$$
\begin{aligned}
y_{I J K} & =\left(Y_{I} Y_{J} Y_{K}\right)^{-1 / 2}\left(\lambda_{I J K}+\frac{\lambda_{I J K}^{\prime}}{e^{\left(q_{I}+q_{J}+q_{K}\right) \pi k T}}\right) \\
M_{a} & =\frac{F^{T}}{2 R}+\frac{1}{2} g_{a}^{2}\left(\frac{\partial \omega_{a}}{\partial Z} F^{Z}+\frac{\partial \omega_{a}^{\prime}}{\partial Z^{\prime}} F^{Z^{\prime}}\right) \\
m_{I \bar{J}}^{2} & =\left(Y_{I} Y_{J}\right)^{-1 / 2}\left[\frac{\pi^{2} q_{I} k \delta_{I J}\left|F^{T}\right|^{2}}{e^{q_{I} \pi k\left(T+T^{*}\right)}-1}+\kappa_{I \bar{J}}\left|F^{Z}\right|^{2}+\frac{\kappa_{I \bar{J}}^{\prime}\left|F^{Z^{\prime}}\right|^{2}}{e^{\left(q_{I} \pi k T+q_{J} \pi k T^{*}\right)}}\right] \\
A_{I J K} & =-\left(Y_{I} Y_{J} Y_{K}\right)^{-1 / 2}\left[F^{T} \frac{\partial}{\partial T} \ln \left(\frac{\lambda_{I J K}+\lambda_{I J K}^{\prime} e^{-\left(q_{I}+q_{J}+q_{K}\right) \pi k T}}{Y_{I} Y_{J} Y_{K}}\right)\right. \\
& \left.\times\left(\lambda_{I J K}+\frac{\lambda_{I J K}^{\prime}}{e^{\left(q_{I}+q_{J}+q_{K}\right) \pi k T}}\right)+F^{Z} \frac{\partial \lambda_{I J K}}{\partial Z}+\frac{F^{Z^{\prime}}}{e^{\left(q_{I}+q_{J}+q_{K}\right) \pi k T}} \frac{\partial \lambda_{I J K}^{\prime}}{\partial Z^{\prime}}\right],
\end{aligned}
$$

where

$$
Y_{I}=\frac{1}{q_{I} k}\left(1-e^{-q_{I} \pi k\left(T+T^{*}\right)}\right),
$$

$g_{a}^{2}$ are $4 \mathrm{D}$ gauge couplings, and $F^{T}, F^{Z}$ and $F^{Z^{\prime}}$ denote the $F$-components of $T, Z$ and $Z^{\prime}$, respectively.

To be more concrete, let us consider the case that both the Yukawa couplings and the brane SUSY breaking come entirely from $\lambda_{I J K}$ and $F^{Z}$ at $y=0$. We further assume that the wavefunctions of $4 \mathrm{D}$ Higgs fields are localized at $y=0$, which would be necessary to generate the top quark Yukawa coupling of order one. Let $Q_{H}$ and $Q_{m}$ denote the Higgs and quark/lepton superfields, respectively, and specify only the quark/lepton flavor indices $(m, n)$ in the Yukawa couplings and trilinear $A$-parameters. We then find

$$
\begin{aligned}
y_{m n} & =\frac{\lambda_{m n}}{\sqrt{Y_{H} Y_{m} Y_{n}}}, \\
M_{a} & =\frac{F^{T}}{2 R}+\frac{1}{2} g_{a}^{2} \frac{\partial \omega_{a}}{\partial Z} F^{Z} \\
m_{m \bar{n}}^{2} & =\frac{1}{\sqrt{Y_{m} Y_{n}}}\left[\frac{\pi^{2} q_{m} k \delta_{m n}}{e^{2 \pi q_{m} R}-1}\left|F^{T}\right|^{2}+\kappa_{m \bar{n}}\left|F^{Z}\right|^{2}\right] \\
A_{m n} & =-y_{m n}\left[F^{T} \frac{\partial}{\partial T} \ln \left(\frac{1}{Y_{H} Y_{m} Y_{n}}\right)+F^{Z} \frac{\partial}{\partial Z} \ln \left(\lambda_{m n}\right)\right]
\end{aligned}
$$


where $\lambda_{m n}$ denote the holomorphic Yukawa couplings in the brane action (2.8) at $y=0$, and

$$
\begin{array}{ll}
\frac{1}{\sqrt{Y_{H}}}=\left(\frac{1}{q_{H} k}\left(1-e^{-q_{H} \pi k\left(T+T^{*}\right)}\right)\right)^{-1 / 2} \approx \sqrt{q_{H} k} & \left(q_{H}>0\right) \\
\frac{1}{\sqrt{Y_{m}}}=\left(\frac{1}{q_{m} k}\left(1-e^{-q_{m} \pi k\left(T+T^{*}\right)}\right)\right)^{-1 / 2} \approx \begin{cases}\sqrt{q_{m} k} & \left(q_{m}>0\right) \\
\sqrt{\left|q_{m}\right| k} e^{-\pi\left|q_{m}\right| k R} & \left(q_{m}<0\right)\end{cases}
\end{array}
$$

As we have noted, $Q_{m}$ with $q_{m}<0$ is localized at $y=\pi$, while $Q_{m}$ with $q_{m}>0$ is localized at $y=0$. As a result, in the case that both the Yukawa couplings and the brane SUSY breaking arise from $y=0$, the Yukawa couplings, $A$-parameters and squark/slepton masses (renormalized at $M_{K K}$ ) involving the light quark/lepton superfields $Q_{m}$ with $q_{m}<0$ are exponentially suppressed as

$$
\begin{aligned}
y_{m n} & =\mathcal{O}\left(e^{-\left(\left|q_{m}\right|+\left|q_{n}\right|\right) \pi k R}\right), \\
A_{m n} & =\mathcal{O}\left(e^{-\left(\left|q_{m}\right|+\left|q_{n}\right|\right) \pi k R} M_{a}\right), \\
m_{m \bar{n}}^{2} & =\mathcal{O}\left(e^{-\left(\left|q_{m}\right|+\left|q_{n}\right|\right) \pi k R} M_{a}^{2}\right),
\end{aligned}
$$

while those involving $Q_{m}$ with $q_{m}>0$ are unsuppressed. On the other hand, there is no localization of $V^{a}$, so no suppression of $M_{a}$. Note that although the squark/slepton masses from $F^{Z}$ are suppressed only for $Q_{m}$ with $q_{m}<0$, the squark/slepton masses from $F^{T}$ are suppressed for all $Q_{m}$ with $q_{m} \neq 0$. This can be easily understood by noting that the radion mediation is equivalent to the Scherk-Schwarz SUSY breaking [8]. The ScherkSchwarz SUSY breaking corresponds to a twist of boundary condition, so its effects are suppressed for any localized mode independently of the position of localization.

In fact, for each model in which both the Yukawa couplings and the brane SUSY breaking arise from $y=0$, there exists a dual model in which the Yukawa couplings and the brane SUSY breaking arise from $y=\pi$. Once we change the sign of all $q_{I}$ of the involved hypermultiplets, the dual model gives the same 4D Yukawa couplings and soft parameters. To see this, let us consider the case that both the Yukawa couplings and the brane SUSY breaking come entirely from $\lambda_{I J K}^{\prime}$ and $F^{Z^{\prime}}$ at $y=\pi$. It is then straightforward to find

$$
\begin{aligned}
y_{m n} & =\frac{e^{-\left(q_{H}+q_{m}+q_{n}\right) \pi k R} \lambda_{m n}^{\prime}}{\sqrt{Y_{H} Y_{m} Y_{n}}}, \\
M_{a} & =\frac{F^{T}}{2 R}+\frac{1}{2} g_{a}^{2} \frac{\partial \omega_{a}^{\prime}}{\partial Z^{\prime}} F^{Z^{\prime}}, \\
m_{m \bar{n}}^{2} & =\frac{1}{\sqrt{Y_{m} Y_{n}}}\left[\frac{\pi^{2} q_{m} k \delta_{m n}}{e^{2 \pi q_{m} R}-1}\left|F^{T}\right|^{2}+\kappa_{m \bar{n}}^{\prime} e^{-\left(q_{m}+q_{n}\right) \pi k R}\left|F^{Z^{\prime}}\right|^{2}\right], \\
A_{m n} & =-y_{m n}\left[F^{T} \frac{\partial}{\partial T} \ln \left(\frac{e^{-\left(q_{H}+q_{m}+q_{n}\right) \pi k T}}{Y_{H} Y_{m} Y_{n}}\right)+F^{Z^{\prime}} \frac{\partial}{\partial Z^{\prime}} \ln \left(\lambda_{m n}^{\prime}\right)\right] .
\end{aligned}
$$

It is rather obvious that these results give (2.14) when $\left(\lambda_{m n}^{\prime}, \kappa_{m \bar{n}}^{\prime}, Z^{\prime}, q_{H}, q_{m}\right)$ are replaced by $\left(\lambda_{m n}, \kappa_{m \bar{n}}, Z,-q_{H},-q_{m}\right)$. So in this case, we need to localize the Higgs fields at $y=\pi$ 
by assuming $q_{H}<0$ in order to have a large top quark Yukawa coupling, and localize the light quark/lepton fields at $y=0$ by assuming $q_{m}>0$ in order to suppress the Yukawa couplings and flavor-violating soft parameters.

The results of (2.14) and (2.17) show that if the brane SUSY breaking takes place only at one of the orbifold fixed points from which the Yukawa couplings arise, the flavorviolating soft parameters of light squark/slepton generations which are induced by $F^{T}$ and one of $F^{Z}, F^{Z^{\prime}}$ are suppressed as (2.16). So, the $\mathrm{AdS}_{5}$ models with localized bulk matter fields can avoid (or at least ameliorate) the SUSY flavor problem while generating the hierarchical Yukawa couplings ${ }^{3}$. Note that all of our results can be applied also to models with flat extra dimension which generate the hierarchical Yukawa couplings by localizing the bulk matter fields. For this, one can simply take the limit $k \rightarrow 0$ while keeping $q_{I} k$ nonzero. In the next section, we will consider the radion-dominated SUSY breaking scenario in this framework, which provides a concrete prediction on soft parameters, and present some models which can pass the constraints on flavor violation without severe fine tuning of parameters.

The results (2.13), (2.14) and (2.17) are obtained from the $4 \mathrm{D}$ effective action (2.9) without including the possible threshold corrections due to massive KK modes. The (exponential) suppression of $y_{m n}$ and $A_{m n}$ for light generations is expected to be stable against KK threshold corrections as it is due to the localization of zero modes in extra dimension. In the $4 \mathrm{D}$ effective SUGRA point of view, the suppression of $y_{m n}$ and $A_{m n}$ is a consequence of an exponentially large Kähler metric $Y_{m \bar{n}} \approx e^{2 \pi\left|q_{m}\right| k R} \delta_{m n} /\left|q_{m}\right| k$ for the case of (2.14), or of an exponentially small holomorphic Yukawa couplings $\tilde{y}_{m n}=e^{-\left(\left|q_{m}\right|+\left|q_{n}\right|\right) \pi k R} \lambda_{m n}^{\prime}$ for the case of (2.17). These features also suggest that the suppressions of $y_{m n}$ and $A_{m n}$ are stable againt KK threshold corrections.

On the other hand, $m_{m \bar{n}}^{2}$ generically get threshold corrections of order $M_{a}^{2} / 8 \pi^{2}$. However the geometric interpretaion for the suppressed flavor-violating soft parameters suggets that the flavor-violating part of the KK threshold correction to $m_{m \bar{n}}^{2}$ is suppressed also by $e^{-\left(\left|q_{m}\right|+\left|q_{n}\right|\right) \pi k R}$ as well as by the loop factor, which means that the KK threshold correction gives just a subleading piece of flavor-violation in $m_{m \bar{n}}^{2}$. There can be additional corrections to soft parameters which are induced by non-renormalizable SUGRA interactions in $4 \mathrm{D}$ effective action [21], but they are suppressed by $M_{K K}^{2} / 8 \pi^{2} M_{P l}^{2} \sim e^{-2 \pi k R} / 8 \pi^{2}$, so are small enough. There are also the model-independent SUSY breaking effects mediated by the $4 \mathrm{D}$ superconformal anomaly [17]. In the models under consideration, the gravitino mass is generically given by $m_{3 / 2}=\mathcal{O}\left(e^{-\pi k R} M_{a}\right)$, so the anomaly-mediated contributions to soft parameters are $\delta M_{a} \sim e^{-\pi k R} M_{a} / 8 \pi^{2}, \delta m_{m \bar{n}}^{2} \sim e^{-2 \pi k R} M_{a}^{2} /\left(8 \pi^{2}\right)^{2}$ and $\delta A_{m n} \sim e^{-\pi k R} M_{a} / 8 \pi^{2}$, which are small enough. So the leading radiative corrections to Yukawa couplings and soft parameters at $M_{W}$ come from the standard renormalization group running down to $M_{W}$ starting from the boundary values at $M_{K K}$ given by (2.14) or (2.17).

We note that the idea of AdS/CFT correspondence suggests a CFT framework which would reproduce the main features of our AdS models. Indeed, models involving super-

\footnotetext{
${ }^{3}$ Another way to ameliorate the SUSY flavor problem using extra dimension has been suggested in [20].
} 
conformal sector have been proposed to generate hierarchical Yukawa couplings as well as exponentially suppressed soft masses [6, 18, 22]. It is then easy to see the correspondence: $q_{I} \pi k R \rightarrow \gamma_{I} \ln \left(\Lambda / M_{c}\right)$, where $\gamma_{I}$ is the anomalous dimension of $Q_{I}$ driven by the coupling to the SC sector, and $\Lambda$ and $M_{c}$ are the cutoff scale and the decoupling scale of the superconformal sector, respectively. The $\mathrm{AdS}_{5}$ models discussed here provide a perturbative framework to generate the hierarchical Yukawa couplings while suppressing the dangerous flavor-violating soft parameters.

\section{Radion-dominated Scenario}

The results of (2.14) and (2.17) show that potentially dangerous flavor-violating soft parameters can be naturally suppressed in $\mathrm{AdS}_{5}$ models. However the resulting soft parameters still involve many adjustable free parameters, particularly in the contributions from the brane SUSY breaking. Obviously, the model becomes much more predictive in the radiondominated scenario 23] which we will discuss in somewhat detail in this section.

To proceed, let us consider the case that the Yukawa couplings come from the brane action at $y=0$ and the Higgs zero modes are localized at $y=0$. To be definite, we assume that the Kaluza-Klein scale is given by $M_{K K}=k e^{-\pi k R} \sim 10^{16} \mathrm{GeV}$, however the results are not sensitive to the precise value of $M_{K K}$. In the radion-dominated scenario for this case, the Yukawa couplings and soft parameters renormalized at $M_{K K}$ are given by

$$
\begin{aligned}
y_{m n} & =\frac{\lambda_{m n} \ln (1 / \epsilon)}{\pi R \sqrt{Y_{H}}} \sqrt{\frac{\phi_{m} \phi_{n}}{\left(1-\epsilon^{2 \phi_{m}}\right)\left(1-\epsilon^{2 \phi_{n}}\right)}}, \\
M_{a} & =\frac{F^{T}}{2 R}, \\
A_{m n} & =2 y_{m n} \ln (1 / \epsilon)\left(\frac{\phi_{m}}{\epsilon^{-2 \phi_{m}}-1}+\frac{\phi_{n}}{\epsilon^{-2 \phi_{n}}-1}+\frac{\phi_{H}}{\epsilon^{-2 \phi_{H}}-1}\right) \frac{F^{T}}{2 R}, \\
m_{m \bar{n}}^{2} & =\delta_{m n}\left(2 \ln (1 / \epsilon) \frac{\phi_{m}}{\epsilon^{\phi_{m}}-\epsilon^{-\phi_{m}}}\left|\frac{F^{T}}{2 R}\right|\right)^{2},
\end{aligned}
$$

where $\epsilon \approx 0.2$ denotes the Cabbibo angle, $Y_{H} \approx 1 / \sqrt{q_{H} k}$, and

$$
\begin{aligned}
& \phi_{m}=\frac{q_{m} \pi k R}{\ln (1 / \epsilon)}=\left(z_{m} c_{m}-\frac{1}{2}\right) \frac{\pi k R}{\ln (1 / \epsilon)}, \\
& \phi_{H}=\frac{q_{H} \pi k R}{\ln (1 / \epsilon)}=\left(z_{H} c_{H}-\frac{1}{2}\right) \frac{\pi k R}{\ln (1 / \epsilon)},
\end{aligned}
$$

for the kink mass $c_{I} k$ and the $Z_{2}$-boundary condition factor $z_{I}(= \pm 1)$ for the $5 \mathrm{D}$ hypermultiplets $\left(H_{I}, H_{I}^{c}\right)$ whose zero modes correspond to the quark/lepton superfields $(I=m)$ or the Higgs superfields $(I=H)$. As we have noted, $c_{I}$ and thus $\phi_{I}$ are likely to be quantized. The observed quark and lepton masses indicate that $\phi_{m}$ are integers for $\epsilon \approx 0.2$, so that all Yukawa couplings are given by an integer power of $\epsilon$ in their order of magnitudes.

Let $\psi_{m}=\left\{q_{i}, u_{i}, d_{i}, \ell_{i}, e_{i}\right\}(i=1,2,3)$ denote the known three generations of the lefthanded quark-doublets $\left(q_{i}\right)$, up-type antiquark-singlets $\left(u_{i}\right)$, down-type antiquark singlets 
$\left(d_{i}\right)$, lepton-doublets $\left(\ell_{i}\right)$, and anti-lepton singlets $\left(e_{i}\right)$. The Yukawa couplings can be written as

$$
\mathcal{L}_{\text {Yukawa }}=y_{i j}^{u} H_{2} q_{i} u_{j}+y_{i j}^{d} H_{1} q_{i} d_{j}+y_{i j}^{\ell} H_{1} \ell_{i} e_{j}
$$

and the squark/sleptons $\phi_{m}=\left\{\tilde{q}_{i}, \tilde{u}_{i}, \tilde{d}_{i}, \tilde{\ell}_{i}, \tilde{e}_{i}\right\}$ have the soft SUSY breaking couplings:

$$
\begin{aligned}
\mathcal{L}_{\text {soft }}= & -\left(A_{i j}^{u} H_{2} \tilde{q}_{i} \tilde{u}_{j}+A_{i j}^{d} H_{1} \tilde{q}_{i} \tilde{d}_{j}+A_{i j}^{\ell} H_{1} \tilde{\ell}_{i} \tilde{e}_{j}\right. \\
& \left.+m_{i \bar{j}}^{2(\tilde{q})} \tilde{q}_{i} \tilde{q}_{j}^{*}+m_{i \bar{j}}^{2(\tilde{u})} \tilde{u}_{i} \tilde{u}_{j}^{*}+m_{i \bar{j}}^{2(\tilde{d})} \tilde{d}_{i} \tilde{d}_{j}^{*}+m_{i \bar{j}}^{2(\tilde{\ell})} \tilde{\ell}_{i} \tilde{\ell}_{j}^{*}+m_{i \bar{j}}^{2(\tilde{e})} \tilde{e}_{i} \tilde{e}_{j}^{*}\right)
\end{aligned}
$$

Let us first consider the quark/squark sector. There can be several different choices of $\phi_{m} \equiv \phi\left(Q_{m}\right)$ which would yield the observed quark masses and mixing angles [24]. Here we will consider one example:

$$
\begin{aligned}
& \phi\left(H_{1}\right)=\phi\left(H_{2}\right)=2, \\
& \phi\left(q_{i}\right)=(-3,-2,2), \\
& \phi\left(u_{i}\right)=(-5,-2,2), \\
& \phi\left(d_{i}\right)=(-3,-2,-2),
\end{aligned}
$$

for which $\tan \beta \sim \epsilon^{2} m_{t} / m_{b}$ is not large. These values of $\phi\left(Q_{m}\right)$ give the following forms of Yukawa coupling matrices

$$
\begin{aligned}
& y_{i j}^{u}=\left(\begin{array}{ccc}
\epsilon^{8} \lambda_{11}^{u} & \epsilon^{5} \lambda_{12}^{u} & \epsilon^{3} \lambda_{13}^{u} \\
\epsilon^{7} \lambda_{21}^{u} & \epsilon^{4} \lambda_{22}^{u} & \epsilon^{2} \lambda_{23}^{u} \\
\epsilon^{5} \lambda_{31}^{u} & \epsilon^{2} \lambda_{32}^{u} & \lambda_{33}^{u}
\end{array}\right), \\
& y_{i j}^{d}=\left(\begin{array}{lll}
\epsilon^{6} \lambda_{11}^{d} & \epsilon^{5} \lambda_{12}^{d} & \epsilon^{5} \lambda_{13}^{d} \\
\epsilon^{5} \lambda_{21}^{d} & \epsilon^{4} \lambda_{22}^{d} & \epsilon^{4} \lambda_{23}^{d} \\
\epsilon^{3} \lambda_{31}^{d} & \epsilon^{2} \lambda_{32}^{d} & \epsilon^{2} \lambda_{33}^{d}
\end{array}\right),
\end{aligned}
$$

where $\lambda_{i j}^{u}$ and $\lambda_{i j}^{d}$ are the coefficients of order unity ${ }^{4}$. The soft parameters renormalized at $M_{K K}$ are determined to be ${ }^{5}$

$$
\begin{gathered}
\frac{A_{i j}^{u}}{y_{i j}^{u}}=2 M_{1 / 2} \ln 5\left(\begin{array}{ccc}
8 & 5 & 3 \\
7 & 4 & 2 \\
5 & 2 & \mathcal{O}\left(\epsilon^{4}\right)
\end{array}\right), \\
\frac{A_{i j}^{d}}{y_{i j}^{d}}=2 M_{1 / 2} \ln 5\left(\begin{array}{ccc}
6 & 5 & 5 \\
5 & 4 & 4 \\
3 & 2 & 2
\end{array}\right), \\
m_{i \bar{j}}^{2(\tilde{q})}=(2 \ln 5)^{2}\left|M_{1 / 2}\right|^{2}\left(\begin{array}{ccc}
9 \epsilon^{6} & 0 & 0 \\
0 & 4 \epsilon^{4} & 0 \\
0 & 0 & 4 \epsilon^{4}
\end{array}\right)
\end{gathered}
$$

\footnotetext{
${ }^{4}$ Here $\lambda_{i j}^{u}$ and $\lambda_{i j}^{d}$ are redefined from $\lambda_{m n}$ in (3.1) to include the coefficients of order one depending on $\ln (1 / \epsilon) / \pi R, \phi\left(q_{i}\right), \phi\left(u_{i}\right)$, and $\phi\left(d_{i}\right)$.

${ }^{5}$ Here we have ignored small corrections suppressed by high powers of $\epsilon$.
} 


$$
\begin{aligned}
& \approx\left|M_{1 / 2}\right|^{2}\left(\begin{array}{ccc}
6 \times 10^{-3} & 0 & 0 \\
0 & 6 \times 10^{-2} & 0 \\
0 & 0 & 6 \times 10^{-2}
\end{array}\right) \\
m_{i \bar{j}}^{2(\tilde{u})} & =(2 \ln 5)^{2}\left|M_{1 / 2}\right|^{2}\left(\begin{array}{ccc}
25 \epsilon^{10} & 0 & 0 \\
0 & 4 \epsilon^{4} & 0 \\
0 & 0 & 4 \epsilon^{4}
\end{array}\right) \\
& \approx\left|M_{1 / 2}\right|^{2}\left(\begin{array}{ccc}
3 \times 10^{-5} & 0 & 0 \\
0 & 6 \times 10^{-2} & 0 \\
0 & 0 & 6 \times 10^{-2}
\end{array}\right) \\
m_{i \bar{j}}^{2(\tilde{d})} & =(2 \ln 5)^{2}\left|M_{1 / 2}\right|^{2}\left(\begin{array}{ccc}
9 \epsilon^{6} & 0 & \\
0 & 4 \epsilon^{4} & 0 \\
0 & 0 & 4 \epsilon^{4}
\end{array}\right) \\
& \approx\left|M_{1 / 2}\right|^{2}\left(\begin{array}{ccc}
6 \times 10^{-3} & 0 & 0 \\
0 & 6 \times 10^{-2} & 0 \\
0 & 0 & 6 \times 10^{-2}
\end{array}\right)
\end{aligned}
$$

where $M_{1 / 2}=F^{T} / 2 R$ denotes the universal gaugino mass at $M_{K K}$. The above Yukawa coupling matrices produce well the observed quark masses and mixing angles. Also, after taking into account the renormalization group evolution from $M_{K K}$ to the weak scale $M_{W}$, the resulting soft parameters pass all phenomenological constraints (including those from flavor-changing processes) for a reasonable range of $M_{1 / 2}$, e.g. $M_{1 / 2} \gtrsim 300 \mathrm{GeV}$, and for generic values of $\lambda_{i j}^{u}$ and $\lambda_{i j}^{d}$ which are taken to be of order unity [19].

One distinctive feature of the radion-mediated SUSY breaking (for dynamically localized matter superfields) is that it gives a rather large value of non-universal $A_{m n} / y_{m n}$ which is of the order of $M_{1 / 2} \ln y_{m n}$. As a consequence, the most stringent constraint on the model comes from $\mu \rightarrow e \gamma$ which would be induced by the slepton $A$-coupling: $A_{i j}^{\ell} H_{1} \tilde{\ell}_{i} \tilde{e}_{j}$. To satisfy the experimental bound on $\operatorname{Br}(\mu \rightarrow e \gamma)$ for generic models of radionmediation, one would need some degree of fine tuning for some of the off-diagonal elements of the lepton Yukawa matrix unless the slepton and gaugino masses are heavier than $\mathrm{O}(1)$ $\mathrm{TeV}$. Another distinctive feature of radion-mediation is that although $A_{m n} / y_{m n}$ are highly flavor-dependent, they are quantized up to small corrections being a high power of $\epsilon$ (see Eq. (3.1). This feature provides a natural way to minimizes the $\mu \rightarrow e \gamma$ rate, which is to choose

$$
\phi\left(\ell_{1}\right)=\phi\left(\ell_{2}\right) \quad \text { or } \quad \phi\left(e_{1}\right)=\phi\left(e_{2}\right)
$$

Let us thus consider the following two examples for the lepton/slepton sector:

$$
\begin{array}{cl}
\text { Model I : } & \phi\left(\ell_{i}\right)=(-3,-3,-1), \\
& \phi\left(e_{i}\right)=(-4,-1,-1), \\
\text { Model II : } & \phi\left(\ell_{i}\right)=(-5,-2,-1), \\
& \phi\left(e_{i}\right)=(-2,-2,-1) .
\end{array}
$$


The model I gives at $M_{K K}$ :

$$
\begin{aligned}
y_{i j}^{\ell} & =\left(\begin{array}{ccc}
\epsilon^{7} \lambda_{11}^{\ell} & \epsilon^{4} \lambda_{12}^{\ell} & \epsilon^{4} \lambda_{13}^{\ell} \\
\epsilon^{7} \lambda_{21}^{\ell} & \epsilon^{4} \lambda_{22}^{\ell} & \epsilon^{4} \lambda_{23}^{\ell} \\
\epsilon^{5} \lambda_{31}^{\ell} & \epsilon^{2} \lambda_{32}^{\ell} & \epsilon^{2} \lambda_{33}^{\ell}
\end{array}\right), \\
\frac{A_{i j}^{\ell}}{y_{i j}^{\ell}} & =2 M_{1 / 2} \ln 5\left(\begin{array}{ccc}
7 & 4 & 4 \\
7 & 4 & 4 \\
5 & 2 & 2
\end{array}\right), \\
m_{i \bar{j}}^{2(\tilde{\ell})} & =(2 \ln 5)^{2}\left|M_{1 / 2}\right|^{2}\left(\begin{array}{ccc}
9 \epsilon^{6} & 0 & 0 \\
0 & 9 \epsilon^{6} & 0 \\
0 & 0 & \epsilon^{2}
\end{array}\right) \\
& \approx\left|M_{1 / 2}\right|^{2}\left(\begin{array}{ccc}
6 \times 10^{-3} & 0 & 0 \\
0 & 6 \times 10^{-3} & 0 \\
0 & 0 & 0.4
\end{array}\right) \\
m_{i \bar{j}}^{2(\tilde{e})} & =(2 \ln 5)^{2}\left|M_{1 / 2}\right|^{2}\left(\begin{array}{ccc}
16 \epsilon^{8} & 0 & 0 \\
0 & \epsilon^{2} & 0 \\
0 & 0 & \epsilon^{2}
\end{array}\right) \\
& \approx\left|M_{1 / 2}\right|^{2}\left(\begin{array}{ccc}
4 \times 10^{-4} & 0 & 0 \\
0 & 0.4 & 0 \\
0 & 0 & 0.4
\end{array}\right)
\end{aligned}
$$

while the model II gives

$$
\begin{aligned}
y_{i j}^{\ell} & =\left(\begin{array}{ccc}
\epsilon^{7} \lambda_{11}^{\ell} & \epsilon^{7} \lambda_{12}^{\ell} & \epsilon^{6} \lambda_{13}^{\ell} \\
\epsilon^{4} \lambda_{21}^{\ell} & \epsilon^{4} \lambda_{22}^{\ell} & \epsilon^{3} \lambda_{23}^{\ell} \\
\epsilon^{3} \lambda_{31}^{\ell} & \epsilon^{3} \lambda_{32}^{\ell} & \epsilon^{2} \lambda_{33}^{\ell}
\end{array}\right), \\
\frac{A_{i j}^{\ell}}{y_{i j}^{\ell}} & =2 M_{1 / 2} \ln 5\left(\begin{array}{lll}
7 & 7 & 6 \\
4 & 4 & 3 \\
3 & 3 & 2
\end{array}\right), \\
m_{i \bar{j}}^{2(\tilde{\ell})} & =(2 \ln 5)^{2}\left|M_{1 / 2}\right|^{2}\left(\begin{array}{ccc}
25 \epsilon^{10} & 0 & 0 \\
0 & 4 \epsilon^{4} & 0 \\
0 & 0 & \epsilon^{2}
\end{array}\right) \\
& \approx\left|M_{1 / 2}\right|^{2}\left(\begin{array}{ccc}
3 \times 10^{-5} & 0 & 0 \\
0 & 6 \times 10^{-2} & 0 \\
0 & 0 & 0.4
\end{array}\right), \\
m_{i \bar{j}}^{2(\tilde{e})} & =(2 \ln 5)^{2}\left|M_{1 / 2}\right|^{2}\left(\begin{array}{ccc}
4 \epsilon^{4} & 0 & 0 \\
0 & 4 \epsilon^{4} & 0 \\
0 & 0 & \epsilon^{2}
\end{array}\right) \\
& \approx\left|M_{1 / 2}\right|^{2}\left(\begin{array}{ccc}
6 \times 10^{-2} & 0 & 0 \\
0 & 6 \times 10^{-2} & 0 \\
0 & 0 & 0.4
\end{array}\right) .
\end{aligned}
$$

The above results pass all phenomenological constraints except for $\mu \rightarrow e \gamma$ when all $\lambda_{i j}^{\ell}$ are taken to be of order unity. To make the models to be consistent with $\mu \rightarrow e \gamma$, one needs some degree of fine tuning for $\lambda_{12}^{\ell}$ and $\lambda_{21}^{\ell}$ defined in (3.9) and (3.10). Through a detailed 
numerical analysis including the renormalization group evolution of soft parameters from $M_{K K} \sim 10^{16} \mathrm{GeV}$ to $M_{W}$, we find that $\lambda_{12}^{\ell}$ and $\lambda_{21}^{\ell}$ are required to satisfy

$$
\begin{aligned}
\text { Model I : } & \lambda_{12}^{\ell} \lesssim 2 \times 10^{-2}\left(\frac{M_{1 / 2}}{500 \mathrm{GeV}}\right)^{2}, \\
& \lambda_{21}^{\ell} \lesssim 10^{-1}\left(\frac{M_{1 / 2}}{500 \mathrm{GeV}}\right)^{2}, \\
\text { Model II : } & \lambda_{12}^{\ell} \lesssim 5 \times 10^{-2}\left(\frac{M_{1 / 2}}{500 \mathrm{GeV}}\right)^{2}, \\
\lambda_{21}^{\ell} & \lesssim 10^{-2}\left(\frac{M_{1 / 2}}{500 \mathrm{GeV}}\right)^{2} .
\end{aligned}
$$

This can be considered to be a fine-tuning of parameters, but not a serious one.

\section{Conclusion}

In this paper, we have examined the soft SUSY breaking parameters in supersymmetric theories on a slice of $\mathrm{AdS}_{5}$ in which the hierarchical Yukawa couplings are generated by localizing the bulk matter fields in extra dimension. In this class of models, the localization of matter zero modes has the common origin as the localization of gravity zero mode, i.e. the gauging of graviphoton which gives nonzero bulk cosmological constant and hypermultiplet masses. As a result, generically the small Yukawa couplings are given by $y \sim e^{-c \pi k R}$ for a constant $c$ of order unity, and so the radion $R$ is required to be stabilized at $e^{-\pi k R} \approx 10^{-2}-10^{-5}$. Unless there is an additional gauge-singlet 5D field other then the minimal 5D SUGRA multiplet, SUSY breaking is mediated by the radion superfield in bulk, and/or some brane superfields at $y=0$ and $\pi$, and/or the 4D SUGRA multiplet in bulk which participates always in SUSY breaking through the conformal anomaly. Although it depends on the details of the radion stabilization, generically the anomalymediated gaugino mass in the models under consideration is suppressed by a small warp factor (compared to to the radion-mediated gaugino mass) as well as by the small loop factor, so can be ignored. We then found that if the brane SUSY breaking takes place only at one of the orbifold fixed points from which the Yukawa couplings arise, which is a natural setting, the dangerous flavor-violating soft parameters can be naturally suppressed by some powers of the warp factor, so the model can avoid (or ameliorate) the SUSY flavor problem. This type of models can be considered as the AdS dual of the recently studied 4D models containing a supersymmetric CFT to generate hierarchical Yukawa couplings. If SUSY breaking is dominated by the radion-mediation, the model provides a concrete prediction on soft parameters which can be tested by low energy experiments. We have presented some models in this framework which pass all phenomenological constraints from flavor violating processes without severe fine tuning of parameters.

\section{Acknowledgments}

KC, DYK and IWK are supported by KRF PBRG 2002-070-C00022 and TK is supported by the Grants-in-Aid for the Promotion of Science No. 14540256 from the Japan Society for the Promotion of Science. 


\section{References}

[1] L. Randall and R. Sundrum, A large mass hierarchy from a small extra dimension, Phys. Rev. Lett. 83 (1999) 3370.

[2] T. Gherghetta and A. Pomarol, Bulk fields and supersymmetry in a slice of AdS, Nucl. Phys. B $586(2000) 141$.

[3] S. J. Huber and Q. Shafi, Fermion masses, mixings and proton decay in a Randall-Sundrum model, Phys. Lett. B 498 (2001) 256.

[4] Y. Grossman and M. Neubert, Neutrino masses and mixings in non-factorizable geometry, Phys. Lett. B 474 (2000) 361.

[5] This subject has been discussed briefly in D. Marti and A. Pomarol, Supersymmetric theories with compact extra dimensions in $N=1$ superfields, Phys. Rev. D 64 (2001) 105025.

K. Choi, Do Young Kim, Ian-Woo Kim and T. Kobayashi, SUSY flavor problem and warped geometry, hep-ph/0301131.

SUSY breaking in warped geometry in different context has been discussed in Z. Chacko and E. Ponton, Bulk gauge fields in warped space and localized supersymmetry breaking, hep-ph/0301171; L. J. Hall, Y. Nomura, T. Okui and S. J. Oliver, Explicit supersymmetry breaking on boundaries of warped extra dimensions, hep-th/0302192.

[6] A. E. Nelson and M. J. Strassler, Suppressing flavor anarchy, J. High Energy Phys. 0009 (2000) 030;

Exact results for supersymmetric renormalization and the supersymmetric flavor problem, $J$. High Energy Phys. 0207 (2002) 021;

T. Kobayashi and H. Terao, Sfermion masses in Nelson-Strassler type of models: SUSY standard models coupled with SCFTs, Phys. Rev. D 64 (2001) 075003;

T. Kobayashi, H. Nakano and H. Terao, Yukawa hierarchy transfer from superconformal sector and degenerate sfermion masses, Phys. Rev. D 65 (2002) 015006;

T. Kobayashi, H. Nakano, T. Noguchi and H. Terao, Sfermion mass degeneracy,

superconformal dynamics and supersymmetric grand unified theories, Phys. Rev. D 66 (2002) 095011:

Yukawa hierarchy transfer based on superconformal dynamics and geometrical realization in string models, hep-ph/0205071.

[7] D. Marti and A. Pomarol, Supersymmetric theories with compact extra dimensions in $N=1$ superfields, Phys. Rev. D 64 (2001) 105025.

[8] D. E. Kaplan and N. Weiner, Radion mediated supersymmetry breaking as a Scherk-Schwarz theory, hep-ph/0108001;

G. von Gersdorff and M. Quiros, Supersymmetry breaking on orbifolds from Wilson lines, Phys. Rev. D 65 (2002) 064016.

[9] A. Ceresole and G. Dall'Agata, General matter coupled $N=2, D=5$ gauged supergravity, Nucl. Phys. B 585 (2000) 143.

[10] R. Altendorfer, J. Bagger and D. Nemeschansky, Supersymmetric Randall-Sundrum scenario, Phys. Rev. D 63 (2001) 125025;

A. Falkowski, Z. Lalak and S. Pokorski, Supersymmetrizing branes with bulk in five-dimensional supergravity, Phys. Lett. B 491 (2000) 172;

J. Bagger, D. Nemeschansky and R. J. Zhang, Supersymmetric radion in the Randall-Sundrum scenario, J. High Energy Phys. 0108 (2001) 057; 
A. Falkowski, Z. Lalak and S. Pokorski, Four dimensional supergravities from five dimensional brane worlds, Nucl. Phys. B 613 (2001) 189.

[11] K. Choi, H. D. Kim and I.-W. Kim, Gauge coupling renormalization in orbifold field theories, J. High Energy Phys. 0211 (2002) 033;

K. Choi, H. D. Kim and I.-W. Kim, Radius dependent gauge unification in AdS(5), J. High Energy Phys. 0303 (2003) 034 ;

K. Choi and I.-W. Kim, One loop gauge couplings in AdS(5), Phys. Rev. D 67 (2003) 045005.

[12] N. Arkani-Hamed, T. Gregoire and J. Wacker, Higher dimensional supersymmetry in $4 D$ superspace, J. High Energy Phys. 0203 (2002) 055.

[13] W. D. Linch, M. A. Luty and J. Phillips, Five dimensional supergravity in $N=1$ superspace, hep-th/0209060.

[14] N. Arkani-Hamed and M. Schmaltz, Hierarchies without symmetries from extra dimensions, Phys. Rev. D 61 (2000) 033005;

E. A. Mirabelli and M. Schmaltz, Yukawa hierarchies from split fermions in extra dimensions, Phys. Rev. D 61 (2000) 113011;

D. E. Kaplan and T. M. Tait, New tools for fermion masses from extra dimensions, J. High Energy Phys. 0111 (2001) 051;

M. Kakizaki and M. Yamaguchi, Proton decay, fermion masses and texture from extra dimensions in SUSY GUTs, hep-ph/0110266;

N. Haba and N. Maru, (S)fermion masses in fat brane scenario, Phys. Rev. D 66 (2002)

055005 A. Hebecker and J. March-Russell, The flavour hierarchy and see-saw neutrinos from bulk masses in 5d orbifold GUTs, Phys. Lett. B 541 (2002) 338

[15] J. A. Casas, J. R. Espinosa and I. Navarro, Unconventional low-energy SUSY from warped geometry, Nucl. Phys. B 620 (2002) 195

[16] A. Hebecker, 5D super Yang-Mills theory in 4-D superspace, superfield brane operators, and applications to orbifold GUTs, Nucl. Phys. B 632 (2002) 101

[17] L. Randall and R. Sundrum, Out of this world supersymmetry breaking, Nucl. Phys. B 557 (1999) 79;

G. F. Giudice, M. A. Luty, H. Murayama and R. Rattazzi, Gaugino mass without singlets, J. High Energy Phys. 9812 (1998) 027

[18] M. A. Luty and R. Sundrum, Supersymmetry breaking and composite extra dimensions, Phys. Rev. D 65 (2002) 066004;

Anomaly mediated supersymmetry breaking in four dimensions, naturally, hep-th/0111231.

[19] K. Choi, D.Y. Kim, W.Y. Song, in preparation.

[20] J. Kubo and H. Terao, Suppressing FCNC and CP-violating phases by extra dimensions, Phys. Rev. D 66 (2002) 116003 ;

K. S. Choi, K. Y. Choi and J. E. Kim, Softening of SUSY flavor problem from asymptotically free bulk interactions, hep-ph/0212354.

[21] K. Choi, J. S. Lee and C. Munoz, Supergravity radiative effects on soft terms and the mu term, Phys. Rev. Lett. 80 (1998) 3686.

[22] A. Karch, T. Kobayashi, J. Kubo and G. Zoupanos, Infrared behavior of softly broken SQCD and its dual, Phys. Lett. B 441 (1998) 235.

[23] Z. Chacko and M. A. Luty, JHEP 0105 (2001) 067 [arXiv:hep-ph/0008103]. 
[24] E. J. Chun and A. Lukas, Quark and lepton mass matrices from horizontal U(1) symmetry, Phys. Lett. B 387 (1996) 99;

K. Choi, E. J. Chun and H. D. Kim, Supersymmetry hierarchy problems and anomalous horizontal U(1) symmetry, Phys. Lett. B 394 (1997) 89. 